\title{
Topological Properties of the Edge Ergodic Layer in Tokamak Plasma
}

\author{
M. Kobayashi, T. Eich, S. S. Abdullaev, and K. H. Finken
}

\begin{abstract}
A topological property appearing in a tokamak with edge ergodized is a transition from a well-ordered system to a chaotic-fractal one. This transition often creates "nice" looking structures such as the well known Mandelbrot picture. In this paper, we present the visualization of the magnetic field structure observed in the torus experiment for technology oriented research-dynamic ergodic divertor, using an asymptotical mapping method. The effect of such a structure on the important transport issue in fusion devices is discussed.
\end{abstract}

Index Terms-Magnetic field structure, particle transport, scattering chaotic system, tokamak.

A MAGNETIC field structure plays an important role on the transport property of the tokamak plasmas. The structure is determined by the current flowing in the plasma and by the several positioning or shaping coils located around the plasma. The resulting field line structure forms the nested flux surfaces that is responsible for confinement, while at the very edge the field lines are guided to the wall in order to control the particle and heat flux coming out of the confinement region. In the torus experiment for technology oriented research (TEXTOR), the dynamic ergodic divertor (DED) [1] has been proposed as an alternative scheme for transport and exhaust control with the edge ergodic plasma created by the external resonant perturbation field. Due to the overlapping of the neighboring magnetic islands caused by the resonant perturbations, the nested flux surfaces break up, and there appears the stochastic structure at the edge as shown in Fig. 1, which is called the ergodic region. In the ergodic region close to the wall, the field lines are called opened because both ends intersect the wall within certain length (connection length $L_{c}$ ). Such a magnetic structure can be viewed as one of scattering chaotic systems, where the length of the field line from one point of the wall to another is sensitively depends on the initial coordinate on the wall. Here, we present one of the examples of such the magnetic structures observed in the ergodic region of the TEXTOR-DED.

In terms of the transport study, the connection length of the field lines is an important parameter because the particles coming out of the core region are guided to the wall flowing convectively along the field lines and the time $\tau_{\boldsymbol{T}}$, needed for

Manuscript received July 2, 2001

M. Kobayashi is with the Institut für Plasmaphysik, Forschungszentrum Jülich GmbH, Euratom Association, Trilateral Euregio Cluster, Jülich 52425, Germany, and also with the Department of Energy Engineering and Science, Graduate School of Engineering, Nagoya University, Nagoya 464-8603, Japan (e-mail: m.kobayashi@fz-juelich.de).

T. Eich, S. S. Abdullaev, and K. H. Finken are with the Institut für Plasmaphysik, Forschungszentrum Jülich GmbH, Euratom Association, Trilateral Euregio Cluster, Jülich 52425, Germany.

Publisher Item Identifier S 0093-3813(02)03076-X

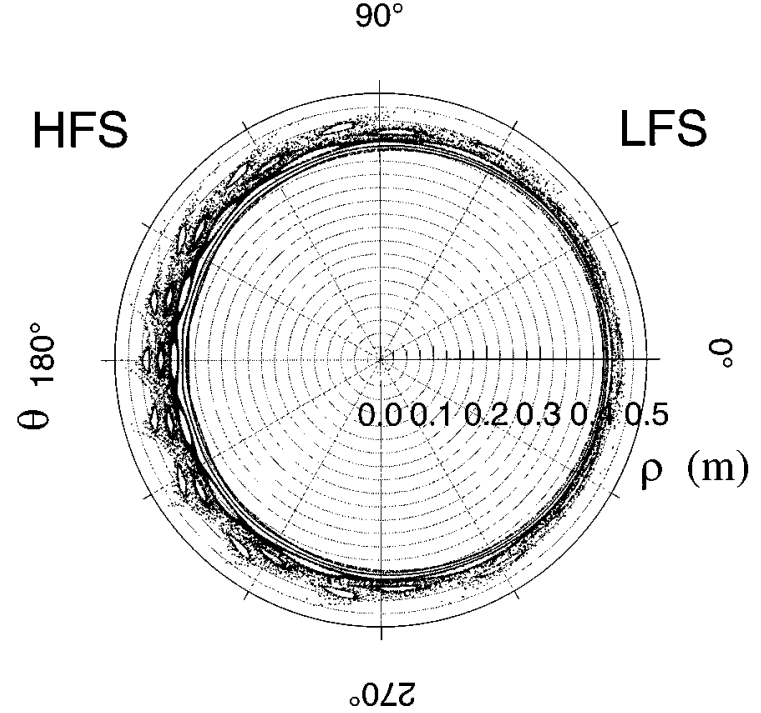

Fig. 1. The Poincaré plot of magnetic field lines in the poloidal cut, where the magnetic structure becomes ergodized due to the perturbation field.

the particles to reach the wall, is estimated as $\tau_{T}=\boldsymbol{L}_{\boldsymbol{c}} / \boldsymbol{v}_{\boldsymbol{p}}\left(\boldsymbol{v}_{\boldsymbol{p}}\right.$ is the flow velocity along the field lines). Since the perturbation coils are installed at the high field side (HFS), most of the field lines intersect the wall at the HFS. In such cases, one finds that there are two locations (cuts) of the highest symmetry, a cut near the low field side (LFS) and another one near the HFS. The structure of the magnetic filed is analyzed based on this picture. Fig. 2 shows the laminar plot [2] at the LFS, where the regions with different connection length are given by the different colors. Using an asymptotical mapping method [3], the field line is traced in both toroidal direction, clockwise, and counter clockwise, starting from the reference plane at the LFS. The connection length is expressed with the number of poloidal turns instead of the length unit, which is indicated at the color bar. In this calculation, the regions are resolved from one to six turns. The green area indicates the three-turn region, and the symmetric twin regions of blue color around $r=44.5 \mathrm{~cm}$ are the two-turn regions. The deep blue area dominating at $r>45 \mathrm{~cm}$ over the whole poloidal direction represents the one-turn region, while at $r<44.5 \mathrm{~cm}$, the area is filled with the long connection length region colored red (more than six turns). Since the mapping method bases on the Hamiltonian system, the area of the each region is preserved over the mappings. Although the pattern of distributing the different poloidal turn regions is rather complicated, we can study the important topological properties from this figure. 


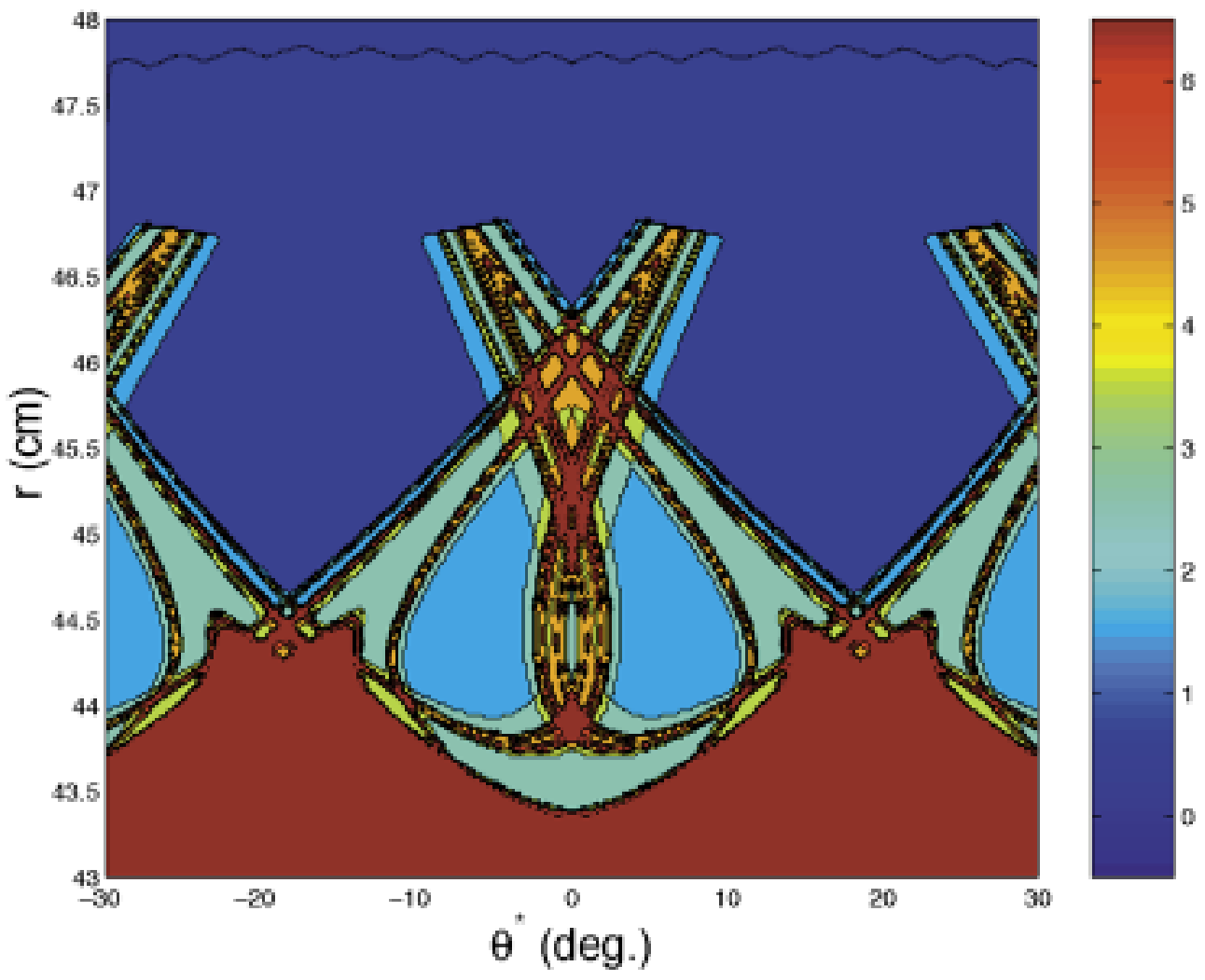

Fig. 2. The laminar plot at the low field side of the TEXTOR-DED, where the regions with different connection length are given by the different colors. The number at the color bar shows the number of poloidal turns experienced by the field line before hitting the wall.

As tracing the field lines, the flux tube experiences the deformation, which is caused by the perturbation field and mostly takes place in front of the perturbation coils. Therefore, the more the field lines make the poloidal turns, the larger the deformation becomes. Due to the area preserving property of the mapping, the deformation gives rise to the indefinite stretching in one direction and compression in another direction. This is clearly observed in the more than six-turn region (red colored). One can find that they are getting thin as going toward the edge, and create the very fine structure being bounded by the one-, two-, three-turn regions. These thin structures are called "finger" after its shape [2]. Such the deformation finally leads to the fractal structure, which is intensively studied in [2] and [4]. From this picture, one should be aware that the particles coming out of the confinement zone along the red region, diffuse easily to the neighboring short connection region (i.e., green, blue, and deep blue regions) through the finger, due to the collision or to the finite Larmor radius, and then led to the wall convectively along the field lines. Now one can find the physics in this description have a lot in common with the standard SOL model [5] that has been well developed for the transport study of the tokamak plasmas. Consequently, it is said that the region with relatively short connection length would play an important role on the transport in the edge ergodic region, where the normal SOL model is still applicable.

In conclusion, the topological properties of the magnetic field in the edge ergodic region have been clearly visualized in the laminar plot. The picture gives the important insights for analyzing the transport at the edge region.

\section{REFERENCES}

[1] K. H. Finken and G. H. Wolf, "Background, motivation, concept and scientific aims for building a dynamic ergodic divertor," Fusion Eng. Des., vol. 37, pp. 337-340, 1997.

[2] T. Eich, D. Reiser, and K. H. Finken, "Two-dimensional modeling approach to transport properties of the TEXTOR-DED laminar zone," Nucl. Fusion, vol. 40, pp. 1757-1772, 2000.

[3] S. S. Abdullaev and K. H. Finken, "Asymptotical and mapping methods in study of ergodic divertor magnetic field in a toroidal system," Phys. Plasmas, vol. 6, pp. 153-174, 1999.

[4] S. S. Abdullaev, Th. Eich, and K. H. Finken, "Fractal structure of the magnetic field in the laminar zone of the Dynamic Ergodic Divertor of the Torus Experiment for Technology-Oriented Research (TEXTOR94)," Phys. Plasmas, vol. 8, pp. 2739-2749, 2001.

[5] P. C. Stangeby, The Plasma Boundary of Magnetic Fusion Devices. London, U.K.: Inst. of Physics, 2000. 